\title{
Characterization And Diversity of The Uterine Microbiota In Simmental And Simbrah Cows By Sequencing The V4 Region of The Gen rRNA-16s During An FTAI Protocol.
}

Emiliano Zapata Luna ( $\nabla$ emiliannoluna2@gmail.com )

Universidad Autonoma de Nuevo Leon Facultad de Medicina Veterinaria y Zootecnia https://orcid.org/0000-0001-6706-9764

Ruben Cervantes Vega

UANL: Universidad Autonoma de Nuevo Leon

\section{Research Article}

Keywords: NGS, beef cows, uterine microbiota, cytobrush, FTAI

Posted Date: July 26th, 2021

DOl: https://doi.org/10.21203/rs.3.rs-708181/v1

License: (c) (1) This work is licensed under a Creative Commons Attribution 4.0 International License. Read Full License 


\section{Abstract}

New generation sequencing techniques allow us to characterize the bacterial populations present in any organ or area. The knowledge of the uterine microbiota in bovines has grown in recent years as it has been shown that it influences fertility, however, there are very few studies in beef cattle. This work aims to contribute by generating information in beef cows, characterizing the uterine microbiome, determined by the sequencing of the hypervariate regions of gen 16S rRNA in samples obtained by the cytobrush technique in open cows prior to and during a standard synchronization, using an intravaginal device of progesterone, plus benzoate of estradiol and prostaglandins. The relative abundance in group I and II were very similar; Firmicutes, Proteobacteria, Bacteroidetes and Actinobacteria. Group III was Firmicutes, Proteobacteria, Bacteroidetes, Actinobacteria, and Fusobacteria, and Group IV, Proteobacteria, Firmicutes, Actinobacteria and Fusobacteria. The taxonomic composition in utero decreases throughout the synchronization protocol. There are internal and external factors that alter bacterial populations such as health status, hormones, pregnancy, medication administration, among others. To establish whether bacterial abundance has an influence on fertility, more robust studies are needed.

\section{Introduction Stating Purpose Of The Work}

Collectively, the microbiome is defined as all microbial genetic material present in a specific environment. Derived from findings from the human microbiome project, it was established that bacteria reside in multiple systems throughout the body contributing vital health functions as part of the normal microbiome. The term "normal" is used instead of "healthy" so that exclusion criteria do not define the biological and clinical criteria of findings in different biological states with the use of technologies based on next-generation sequencing, 16S rRNA gene sequencing and bioanalysis using high-performance computational methods to process and analyze the huge amount of data generated (Peterson et al. 2009).

Uterine disease provides an opportunity to study animal resilience because, despite the exposure of the uterus to similar bacteria, the incidence of diseases varies between species groups, milk production levels, breeds and farms (Santos et al. 2011).

Studies conducted in dairy cows showed a profound shift in absolute and relative abundance in the genus of bacteria present in the postpartum of both healthy cows and cows with metritis, and that intrauterine microbiota is more diverse and complex than described by traditional culture methods (Santos et al. 2011). Using the Illumina MiSeq platform to sequence the 16S rRNA gene was found at the phylum level that Bacteroidetes (28.9), Proteobacteria (22.5), Fusobacterium (20.2), Firmicutes (15.8), Tenericutes (10.7), Actinobacteria (1.0), Spirochaetes (0.5) and Verrucomyrophobia (0.1) were the eight most abundant of the 28 phyla found in the uterus in dairy cows, and that Bacteroidetes were more abundant in cows with metritis than in healthy cows as the abundance of Fusobacteria in cows with metritis and healthy cows was similar (Jeon et al. 2015). 
In contrast, under particular production conditions such as beef cows kept indoors in individual feeders, subclinical metritis has been reported to be an important pathology in Piedmontese beef cows which affects fertility and that the presence of bacteria in the uterus is harmful to fertility, but only when associated with the presence of polymorphonuclear cells (Ricci et al. 2015). A study evaluating the diversity of the bovine vaginal and uterine bacterial community and its relationship to fertility in cows in the calf-cow system where hypervariable regions V1 to V3 of the 16S rRNA gene were sequenced from day 59 to $80 \pm 2.6$ on the postpartum day, using fixed-time insemination and a standard 7-day Co-Synch synchronization protocol, found a decrease in the number of bacterial species over time in the uterus of pregnant and nonpregnant cows, which suggested that the change in bacterial communities in reproductive organs and the diversity of the phylogenetic relationship that leads to conception can contribute to the successful establishment of pregnancy (Ault et al. 2019b)

Some different techniques for microbiological sampling of the uterus are used, such as saline serum flushing samples (Jeon et al. 2015; Ault et al. 2019b) or artificial insemination guns adapted with cytobrush or swab, to collect the intra uterine sample. (Ricci et al. 2015; Cardoso et al 2017; Mitra et al. 2017)

This work aims to contribute by generating information in beef cows, characterizing the uterine microbiome, determined by the sequencing of the hypervariate regions gen 16S rRNA in samples obtained by the cytobrush technique in open cows prior to and during a standard synchronization, using an intravaginal device of progesterone, plus benzoate of estradiol and prostaglandins.

\section{Materials And Methods}

Samples of the uterine endometrium were taken in non-pregnant cows, using cytobrush technique (Vallejo et al. 2014; Mitra et al. 2017), at Simmental and Simbrah cattle ranch, located at latitude N 24.79367 and longitude 0 99.54482, Linares N.L. during January and February 2020. In the study, 38 purebred cows were initially selected (Simmental: 24 Simbrah: 14 ) with 166.5 days postpartum (PPD), the initial inclusion criteria were cows with more than 50 days postpartum and did not show mucopurulent vaginal discharges or were being treated with antibiotics or anti-inflammatory for two weeks prior to the start of the progestogen synchronization program, the assessment of the body condition was $4 \pm 0.54$ SD. Seventeen cows were not included in the final analysis because they did not meet the inclusion criteria, by death or because uterine samples in the laboratory did not meet more than 10,000 sequences. A progestogen synchronization protocol was used for the purpose of performing Fixed Time Artificial Insemination (FTAl), in day - 10 a sample of the uterine mucosa was taken prior to introducing an intravaginal device with $1.9 \mathrm{~g}$ of progesterone, $2 \mathrm{mg}$ oestradiol benzoate was administered via I.M., on day -2 of the protocol the device was removed, a sample of the endometrium was taken and $25 \mathrm{mg}$ of dinoprost trometamina I.M. and 400 U.I. of eCG were administered intramuscularly.

Each cytobrush used for the sampling of the endometrium was introduced in a cryotube and subsequently snap-freezed with liquid nitrogen. To each vial was added $2 \mathrm{ml}$ of ethanol at $96^{\circ} \mathrm{C}$ and 
centrifuged at $1400 \mathrm{rpm}$ by $10 \mathrm{~min}$, stored and sent in a temperature of $78.6^{\circ} \mathrm{C}$ (in dry ice) to Centro de Investigación en Alimentación y Desarrollo (CIAD) in Mazatlan, Sinaloa for DNA extraction, sequencing and bioinformatics analysis.

DNA extraction was performed with a traditional CIAD standardized protocol of Cetil trimethyl ammonium Bromide (CTAB) for microbiological samples (Alejos et al. 2014). To determine the microbiota present in the samples, region 16S-V4 (primers V4-515f and V4-806r) was amplified by PCR with Illumina adapters. Indexes were added to the final products in accordance with the protocol recommended by Illumina. Finally, the samples were quantified in Qubit and mixed into an equimolar pool to be sequenced into Illumina's Mini Seq equipment under standard conditions (300 cycles, 2X150). Samples with low number of sequences in the first run were resequenced.

Biobacter software, which is the bioinformatics server of the CIAD, Mazatlán unit was used for bioinformatics analysis operating with Biolinux 8 operating system (linux Ubuntu 14.04), so that the cleaning of sequences with pair-end_cleaner v1.1.1.1, the elimination of chimeric sequences with chimera_detector v1.5.0 and the classification of the sequences in taxa with mg_classifier v1.8.2.

The data were statistically analyzed using a blocked factorial design $2^{2}$ to evaluate together the effect of the (Group I: PPG 1_Day-10 Prior FTAI) $=$ cows with $\geq 50$ and $\leq 100$ days postpartum during day -10 prior FTAI, (Group II: PPG 2_Day-10 Prior FTAI) = cows than postpartum period $\geq 101$ days and -10 days prior FTAl, (Group III: PPG 1_Day-2 Prior FTAI) = cows with $\geq 50$ and $\leq 100$ days postpartum during the 2 days prior FTAI and (Group IV: PPG 2_Day-2 Prior FTAI) = cows $\geq 101$ days postpartum during the -2 days prior FTAI of a hormonal protocol for the purpose of fixed-time artificial insemination.

\section{Results}

OTU's of 30 phyla, 59 classes, 124 orders, 215 families, 513 genera and 485 genera plus species were identified by the new generation sequencing. At the phylum level, a significant difference was found as the main effect for the factor of days prior to fixed-time artificial insemination (FTAI), for Actinobacteria $(p=0.03)$ and Fusobacterium $(p=0.03)$, in both cases these bacterial phyla are increased two days before FTAI in cows subjected to a protocol with a progesterone intravaginal device, compared to the group of cows 10 days before FTAl and just before the progesterone intravaginal device is inserted. No interaction was found between groups. Bacterial phyla with a higher relative abundance sequenced between Group I and II were very similar; Firmicutes, Proteobacteria, Bacteroidetes and Actinobacteria. The relative bacterial abundance obtained in Group III was Firmicutes, Proteobacteria, Bacteroidetes, Actinobacteria, and Fusobacteria, and for Group IV, the relative bacterial abundance found was Proteobacteria, Firmicutes, Actinobacteria and Fusobacteria. Using the OTU's identified in each group, a heatmap and histogram for relative abundance for phyla were constructed utilizing OTU found in each group (Fig. 1).

Similarly, using the analysis method described, it was determined at the bacterial class's level (Fig. 2). No significant interaction was found between the groups studied. In contrast, as the main effect in the 
postpartum groups evaluated, significant difference was found for the classes Deltaproteobacteria $(\mathrm{p}=$ $0.01)$, Alphaproteobacteria $(p=0.04)$, Campylobacteria $(p=0.04)$ and Anaerolineae $(p=0.05)$, in all these cases more OTU's of the bacterial classes were identified in cows between 50 and 100 days postpartum compared to cows with more than 101 days postpartum. Significant differences were also found as the main effect of the days prior to FTAl factor, as the classes Actinobacteria $(p=0.03)$ and Fusobacteria $(p=$ 0.03 ) were increased in the group of cows used, two days before FTAl in beef cows subjected to a protocol with a progesterone intravaginal device. The relative abundance of the most relevant classes, found for groups I, II, III and IV was Clostridia, Bacteroidias, Gammaproteobacteria, Actinobacteria, Bacilli and Fusobacterias which in groups I and II do not appear, but which rises in relative abundance in groups III and IV.

In bacterial orders, interaction was found between the groups evaluated in the factorial design for the order Kinesporiales (p. 0.05) and Actinomycetales (p. 0.02). Significant differences were found between the postpartum days factor for Myxococcales (p. 0.02), Rhodobacterales (p. 0.03), Cellvibrionales (p. 0.04) and Campylobacterales (p. 0.04) where OTU's were significantly identified in cows between 50 and 100 days compared against those with more than 101 days postpartum and for the factor of days prior to fixed-time artificial insemination significant difference was found for orders; Micrococcales (p. 0.03), Pasteurellales (p. 0.03), Fusobacteriales (p. 0.03) where significant OTU's were determined two days prior to FTAI and Aeromonadales, where there was significance in cows 10 days prior to FTAI compared to cows 2 days before FTAI (p. 0.03),. Orders with the greatest relative abundance were Clostridiales, Bacteroidales, Bacillales and Micrococcales (Fig. 3).

As for the sequenced bacterial families, significant interaction was found for Kineosporiaceae $(p=0.02)$, Promicromonosporaceae $(p=0.03)$ and Actinomycetaceae $(p=0.05)$. In the postpartum group as the main effect, significant differences were found in the families Rhodobacteraceae $(p=0.03)$, Cellvibrionaceae $(p=0.04)$, Christensenellaceae $(p=0.04)$, Campylobacteraceae $(p=0.04)$ and Defluviitaleaceae $(p=0.05)$ where OTU's were significantly identified in cows between 50 and 100 days compared against those with more than 101 postpartum days. In the days leading up to FTAl, significant differences were found for the families Porphyromonadaceae $(p=0.03)$, Pasteurellaceae $(p=0.03)$, Micrococcaceae $(p=0.03)$, Fusobacteriaceae $(p=0.04)$ and Pseudomonadaceae $(p=0.05)$, families in which OTU's were sequenced significantly two days prior to FTAl compared to 10 days prior to FTAl just the day the protocol began, in addition, in the family Succinivibrionaceae OTU's were found significantly on day 10 prior to FTAl, compared to day 2 prior to FTAI $(p=0.02)$. The families with the highest relative abundance are Ruminococcaceae, Lachnospiraceae, Prevotellaceae and Fusobacteriaceae (Fig. 4).

No significant interaction was found between sequenced genus. In the postpartum group as the main effect, significant differences were found in the genera Atopostipes where OTU's were significantly sequenced in cows with less than 100 days postpartum $(p=0.02)$ unlike the Campylobacter $(p=0.04)$ and Negativibacillus ( $p=0.05$ ) where there was a significant difference in cows with more than 101 postpartum days compared against cows between 50 and 100 days postpartum. Similarly, significant difference was found for the genus Porphyromonas $(p=0.03)$, Glutamicibacter $(p=0.03)$, Fusobacterium 
$(p=0.04)$ and Pseudomonas $(p=0.05)$ where it is found were in greater abundance two days before FTAI compared to 10 days before FTAl, in contrast to the genus Ruminobacter where more OTU's were found on day ten prior to FTAl compared to day two prior to FTAI $(p=0.03)$. The genera with the greatest relative abundance they found are Fusobacterium, Acinetobacter, Roseburia and Bacillus (Fig. 5).

Among bacteria sequenced up to genus and species, significant difference in interaction was found for Arcanobacterium hippocoleae $(p=0.05)$ and Trueperella pyogenes $(p=0.05)$, additionally significant differences were found between postpartum groups as the main effect in Brachybacterium sacelli where more was found in cows between 50 and 100 postpartum days than in cows over 101 days postpartum $(p=0.05)$ and for the days leading up to FTAI as the main effect, significant differences were found for Serratia marcescens where more than 10 days prior to FTAI was found $(p=0.05)$ and Glutamicibacter creatinolyticus where more OTU's were sequenced on day 2 prior to FTAI (p. 0.05). The most commonly sequenced genus and species relative abundance are: Fusobacterium necrophorum, Histophilus somni, Moraxella Bovoculi and Glutamicibacter mysorens (Fig. 6)

\section{Diversity by Shannon's index}

Diversity according to the Shannon's index has a mean of $3.30 \pm .71$ SD for cows sampled on day -10 of the protocol. The mean of Shannon's index on day -2 of the protocol is $2.54 \pm .93 \mathrm{SD}$. There is a significant decrease $(p=0.006)$ between days -10 and -2 . (Fig. 7$)$

\section{Discussion}

Our investigation explores a new field in open beef cows during the postpartum breeding period by characterization via new generation sequencing uterine microbiome biomass. Besides the finding of bacterial genera with techniques of cultures on agar in the uterus of beef cows has been published, Ricci (2014) isolated different bacteria such as Trueperella spp, Prevotella spp, Fusobacterium spp, Staphylococcus spp, Streptococcus spp, Mycoplasma spp. However, the difficulty of the growth of aerobic bacteria and bacterial identification makes the characterization of the uterine microbiota by this method unfeasible.

A study in beef cows Ault (2019a) was conducted where they sequenced the hypervariable region V1, V2 and $\mathrm{V} 3$ of the rRNA $16 \mathrm{~s}$ gene in a 21-day insemination protocol using prostaglandin, cows had an average of $80 \pm 2.6$ days postpartum and a decrease in bacterial diversity was found, using the indices of Chao's 1, Faith's and Shannon's between day -21 and day -2 of the protocol regardless of their pregnancy status. Similarly in our study using the Shannon's index, diversity decreases between day - 10 to -2 of a progestogen protocol.

In Ault (2019b) the most abundant filum in both pregnant and non-pregnant cows was firmicutes. In group I, II and III the most abundant filum is firmicutes, however, in group IV the most abundant filum was Proteobacteria. Ruminococcaceae was also the largest family found for both studies, except for group IV. 
Beef cows that have between 50- and 100-days postpartum show a greater number of the orders Myxococcales, Rhodobacterales, Cellvibrionales, Campylobacterales compared to those that were more than 100 days postpartum.

There is the interaction for Arcanobacterium hippocoleae and Trueperella pyogenes, the latter being a potential pathogen producing endometritis. Fertility studies and the interaction of these two species are needed. The taxonomic composition in utero decreases throughout the synchronization protocol. There are internal and external factors that alter bacterial populations such as health status, hormones, pregnancy, medication administration, among others. To establish whether bacterial abundance has an influence on fertility, more robust studies are needed.

\section{Declarations}

\section{(f) Acknowledgements}

I express my gratitude to the Consejo Nacional de Ciencia y. Tecnología (CONACyT) for the scholarship awarded during my Master's Degree.

To the project PROFEXCE 2020 for funding this research.

To the Centro de Investigación y Producción Agropecuaria (CIPA UANL) for welcoming me at their facilities and providing me with the animals for this study.

\section{(g) Statement of Animal Rights}

All animal procedures were performed in accordance with the approved guidelines and regulations, and the ethical approval of the bioethics and animal welfare committee (UANL-Dictamen20/2019).

\section{(h) Conflict of Interest Statement}

The authors have no conflicts of interest to declare.

ZE conducted experiments. ZE and RC wrote the manuscript. RC analyzed data. All authors read and approved the manuscript.

The datasets generated and/or analyzed during the current study are available from the corresponding author on reasonable request.

\section{References}

1. Alejos LP, Aragón MM, Cornejo RA. Extracción y purificación de ADN. 2014. From inecc web site: http://www2.inecc.gob.mx/publicaciones2/libros/710/extraccion.pdf accesed: 06/06/21

2. Ault TB, Clemmons BA, Reese ST, Dantas FG, Franco GA, Smith TPL, et al. Uterine and vaginal bacterial community diversity prior to artificial insemination between pregnant and nonpregnant 
postpartum cows. Journal of Animal Science 2019b;97:4298-4304.

https://doi.org/10.1093/jas/skz210

3. Ault, T. B., Clemmons, B. A., Reese, S. T., Dantas, F. G., Franco, G. A., Smith, T., Edwards, J. L., Myer, P. R., \& Pohler, K. G. (2019a). Bacterial taxonomic composition of the postpartum cow uterus and vagina prior to artificial insemination1. Journal of animal science, 97(10), 4305-4313. https://doi.org/10.1093/jas/skz212

4. Cardoso B., Oliveira, M., Pugliesi, G., Batista, E. and Binelli, M. Cytobrush: A tool for sequential evaluation of gene expression in bovine endometrium. Reproduction in Domestic Animals, 2017;52(6), pp.1153-1157.

5. Jeon SJ, Vieira-Neto, A., Gobikrushanth, M., Daetz, R., Mingoti, R., Parize, A., de Freitas, S., da Costa, A., Bicalho, R., Lima, S., Jeong, K. and Galvão, K. Uterine Microbiota Progression from Calving until Establishment of Metritis in Dairy Cows. Applied and Environmental Microbiology, 2015;81(18), pp.6324

6. Mitra A, Maclntyre DA, Mahajan V, Lee YS, Smith A, Marchesi JR, et al. Comparison of vaginal microbiota sampling techniques: Cytobrush versus swab.Scientific Reports 2017;7(1):1-10.

7. Peterson J, Garges S, Giovanni M, Mclnnes P, Wang L, Schloss JA, et al. The NIH Human Microbiome Project. Genome Research 2009;19:2317-2323. https://doi.org/10.1101/gr.096651.109.

8. Ricci A, Gallo S, Molinaro F, Dondo A, Zoppi S, Vincenti L. Evaluation of subclinical endometritis and consequences on fertility in piedmontese beef cows. Reproduction in Domestic Animals 2015;50:142-148. https://doi.org/10.1111/rda.12465

9. Santos TMA, Gilbert R, Bicalho RC. Metagenomic analysis of the uterine bacterial microbiota in healthy and metritic postpartum dairy cows. Journal of Dairy Science 2011;94:291-302. https://doi.org/10.3168/jds.2010-3668.

10. Vallejo Timarán, D., Chaves Velásquez, C., Astaíza Martínez, J., Benavides Melo, C. and Jurado Ojeda, $X$. Endometritis subclínica diagnosticada mediante cytobrush y comportamiento reproductivo en vacas del municipio de Pupiales, Colombia. Revista de Medicina Veterinaria, 2014;(27), p.111.-6332.

\section{Figures}


A

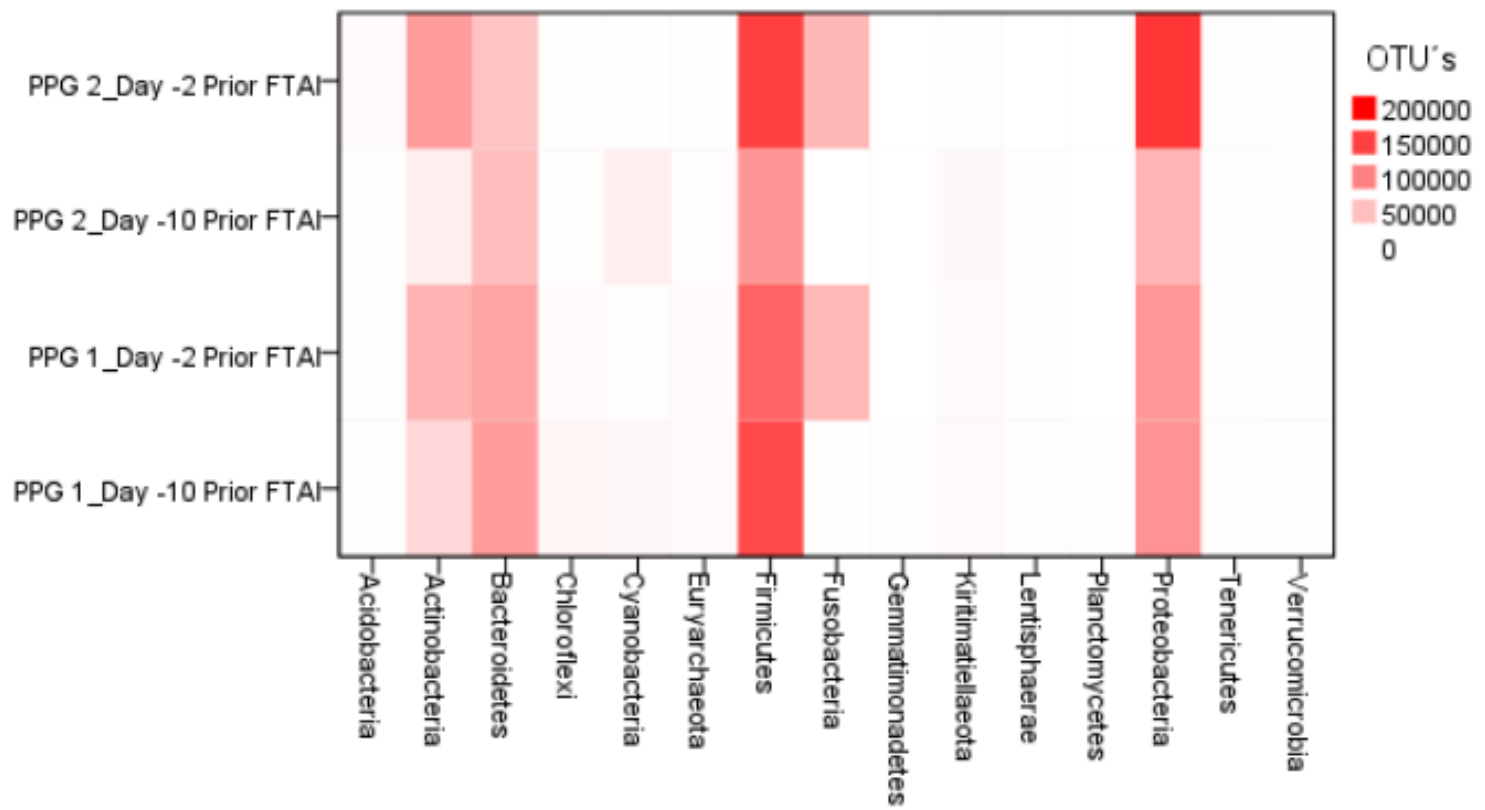

Phylum

B

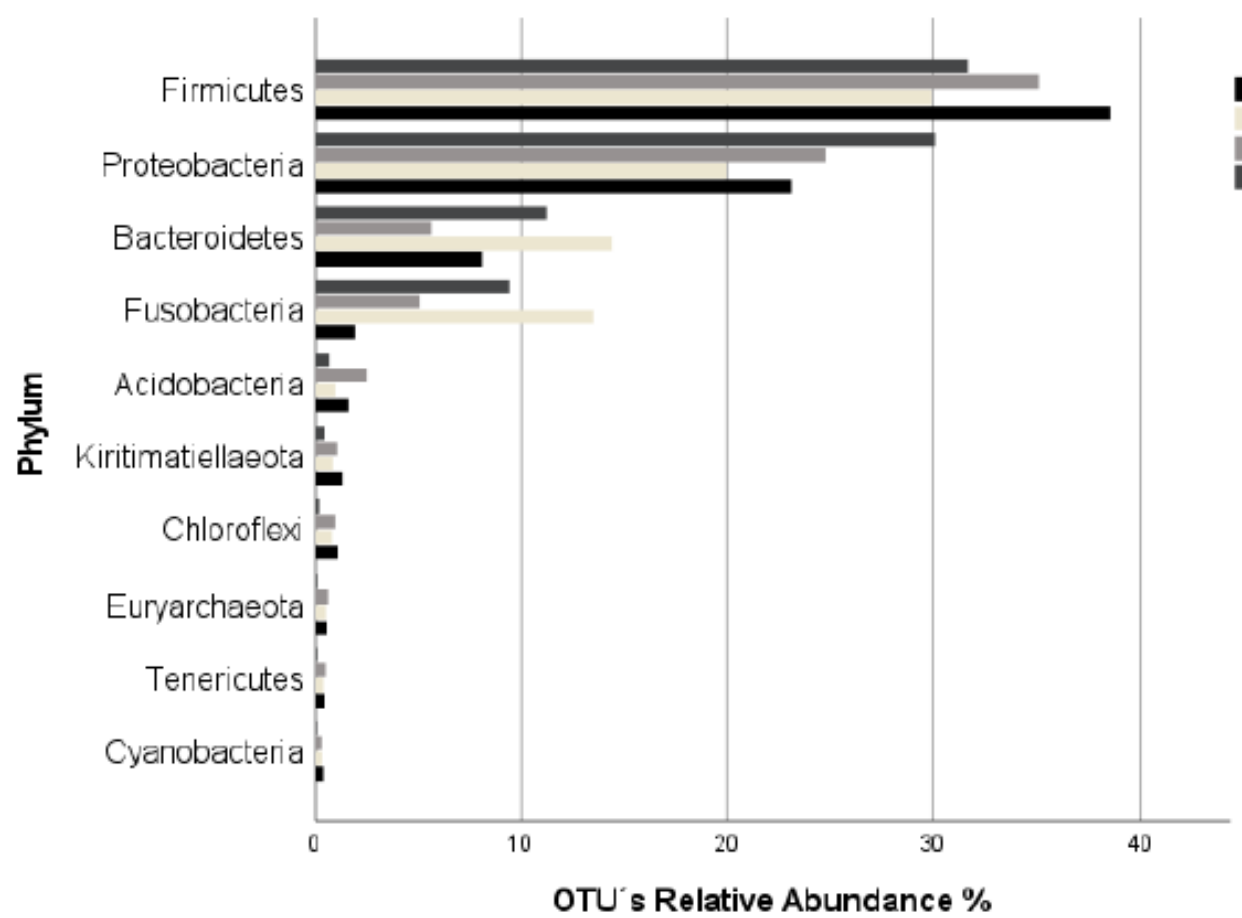

Group

IPPG 1_Day - 10 Prior FTAI

PPG 1 Day -2 Prior FTA

PPG 2-Day - 10 Prior FTAl

PPG 2_Day -2 Prior FTA

\section{Figure 1}

Relevant phyla identified by NGS in beef cow's prior an FTAI. A. Heatmap of relevant phyla for each group, including those significant differences, was determined. B. Percentage of relative abundance of the ten most phyla sequenced ordered by group 
A

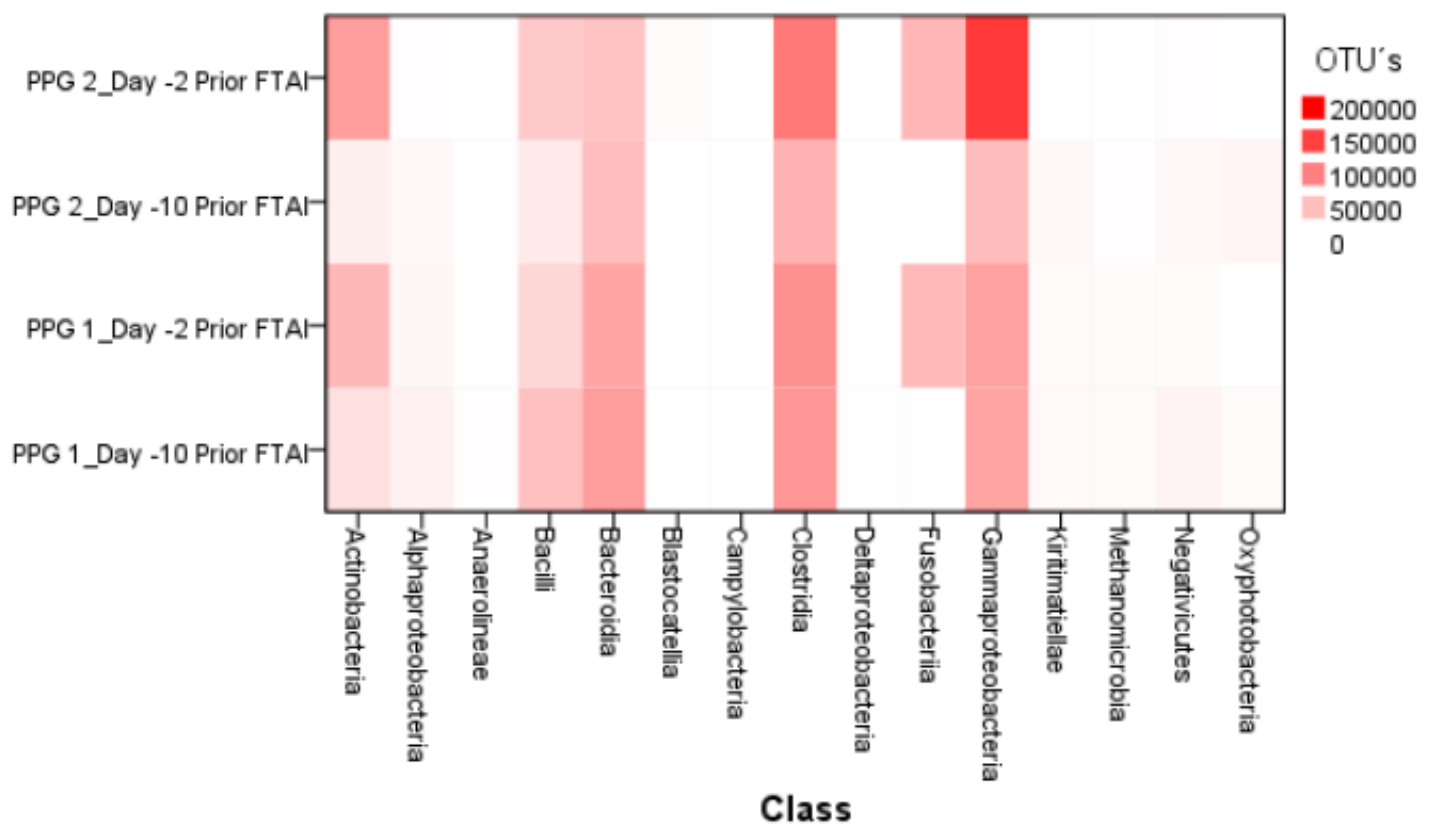

B

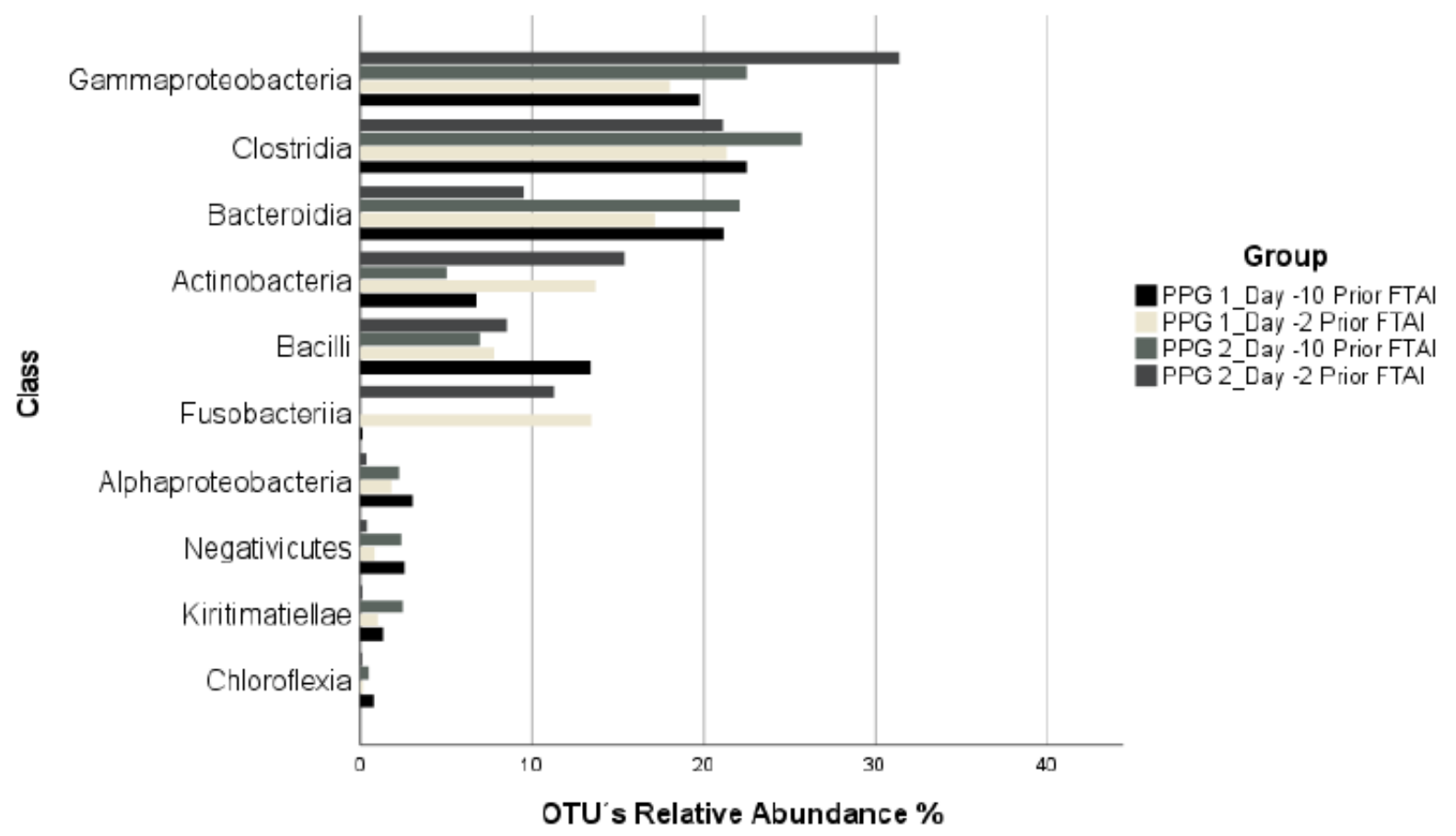

Figure 2

Relevant classes identified by NGS in beef cow's prior an FTAI. A. Heatmap of relevant classes for each group, including those significant difference was determined. B. Percentage of relative abundance of the ten most classes sequenced ordered by group 


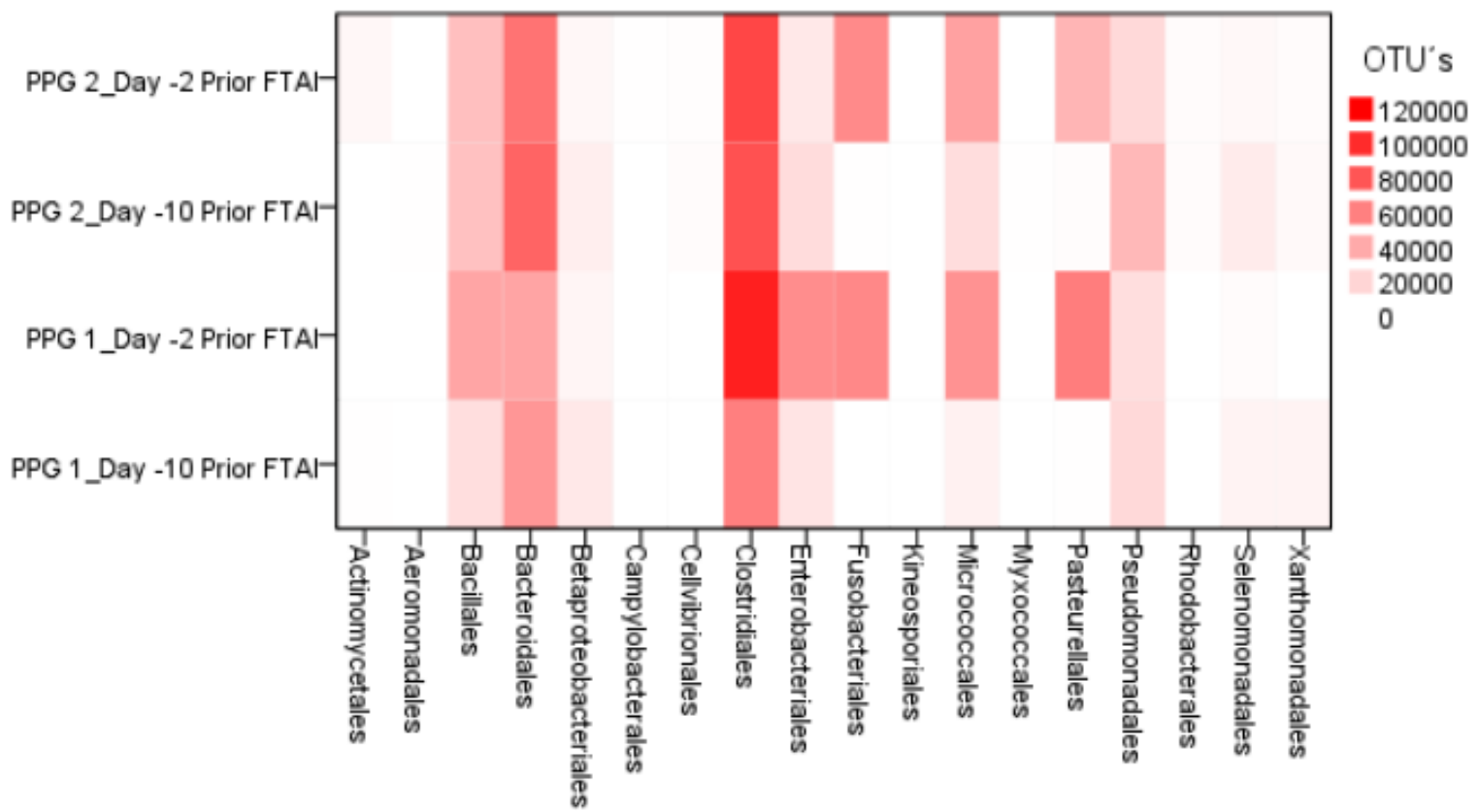

\section{Order}

B

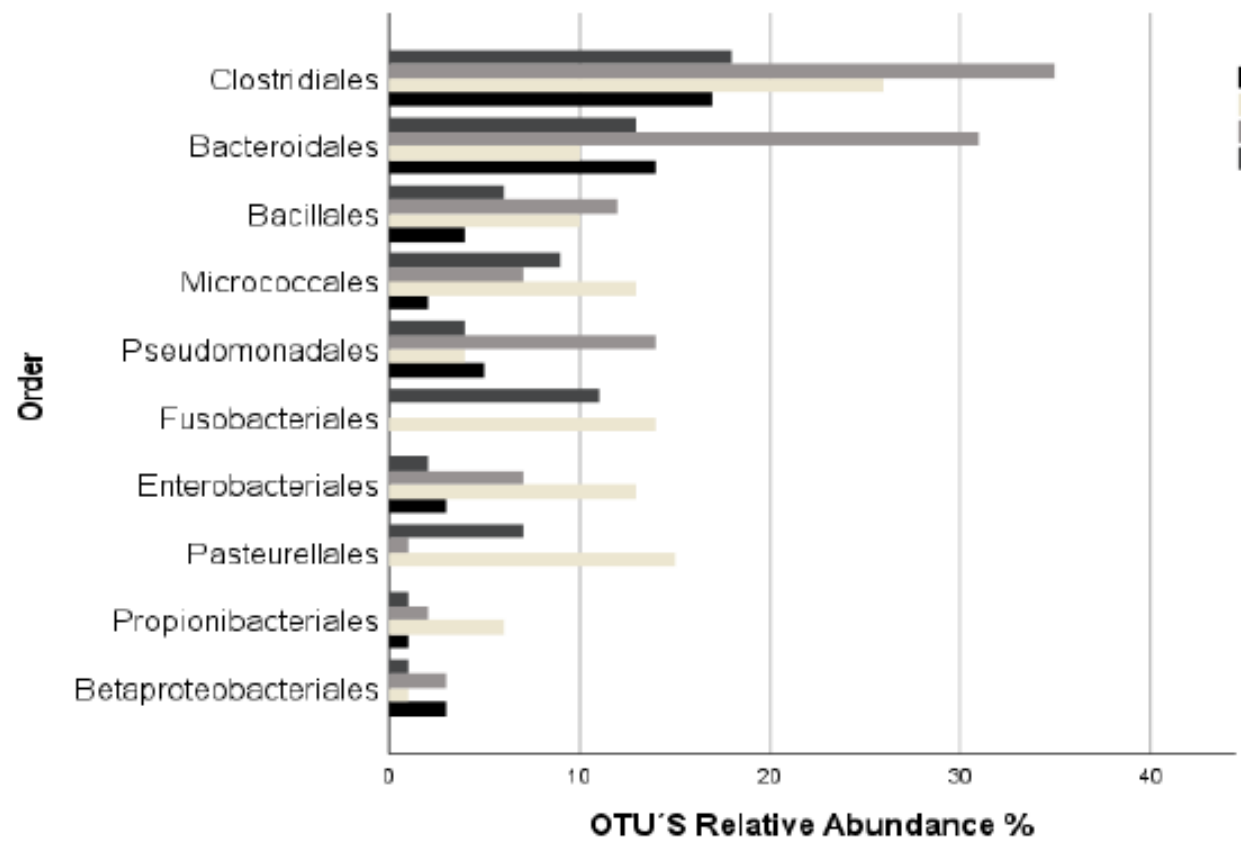

Group

PPG 1_Day -10 Prior FTAI

PPG 1 Day -2 Prior FTAI

PPG 2 Day -10 Prior FTA

PPG 2 Day -2 Prior FTAI

\section{Figure 3}

Relevant orders identified by NGS in beef cow's prior an FTAI. A. Heatmap of relevant orders for each group, including those significant difference was determined. B. Percentage of relative abundance of the ten most orders sequenced ordered by group 


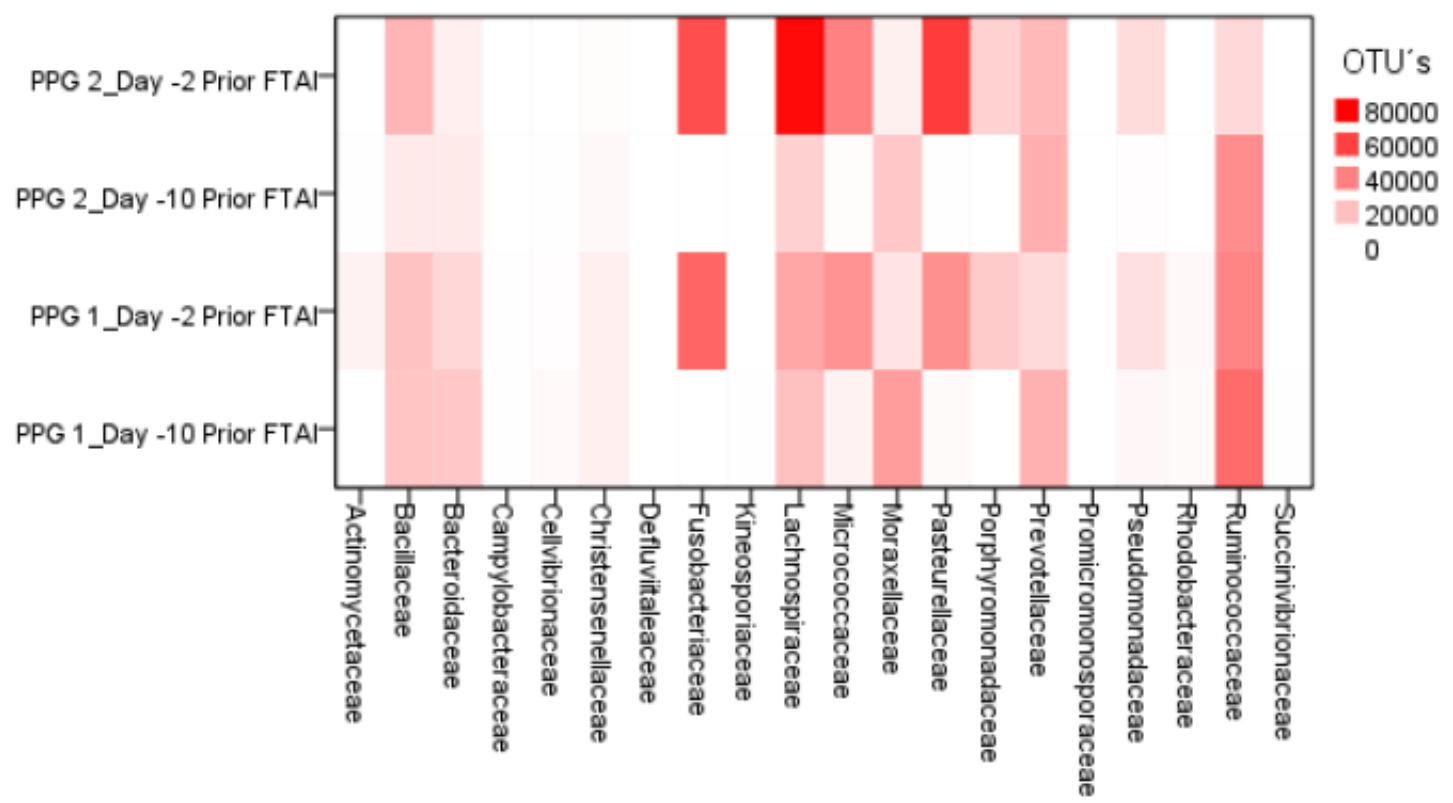

Family

B

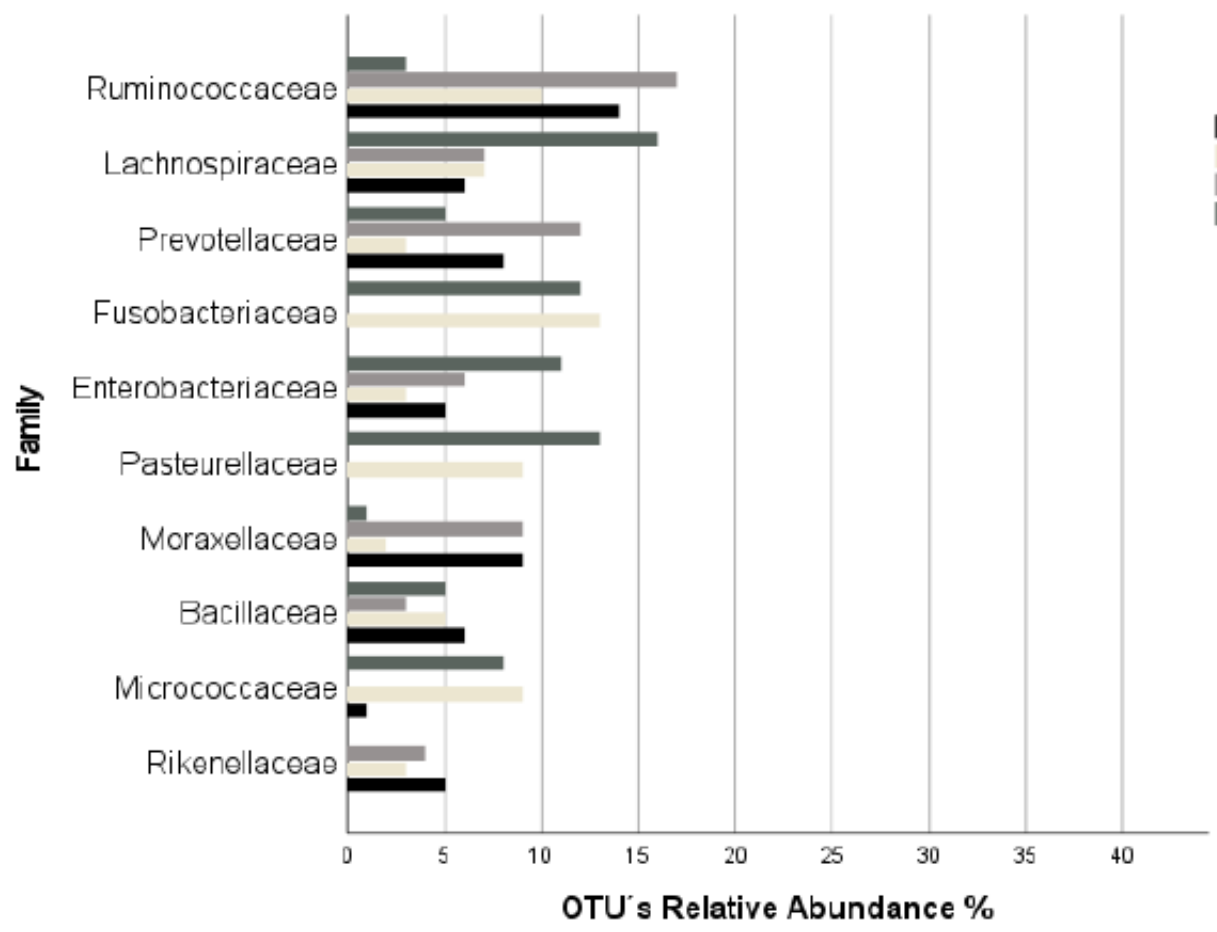

\section{Class}

PPG 1 Day -10 Prior AIFT

PPG 1_Day -2 Prior AIFT PPG 2 Day -10 Prior AIFT DPPG 2_Day -2 Prior AIFT

\section{Figure 4}

Relevant bacterial families identified by NGS in beef cow's prior an FTAI. A. Heatmap of relevant families for each group, including those significant difference was determined. B. Percentage of relative abundance of the ten most families sequenced ordered by group 


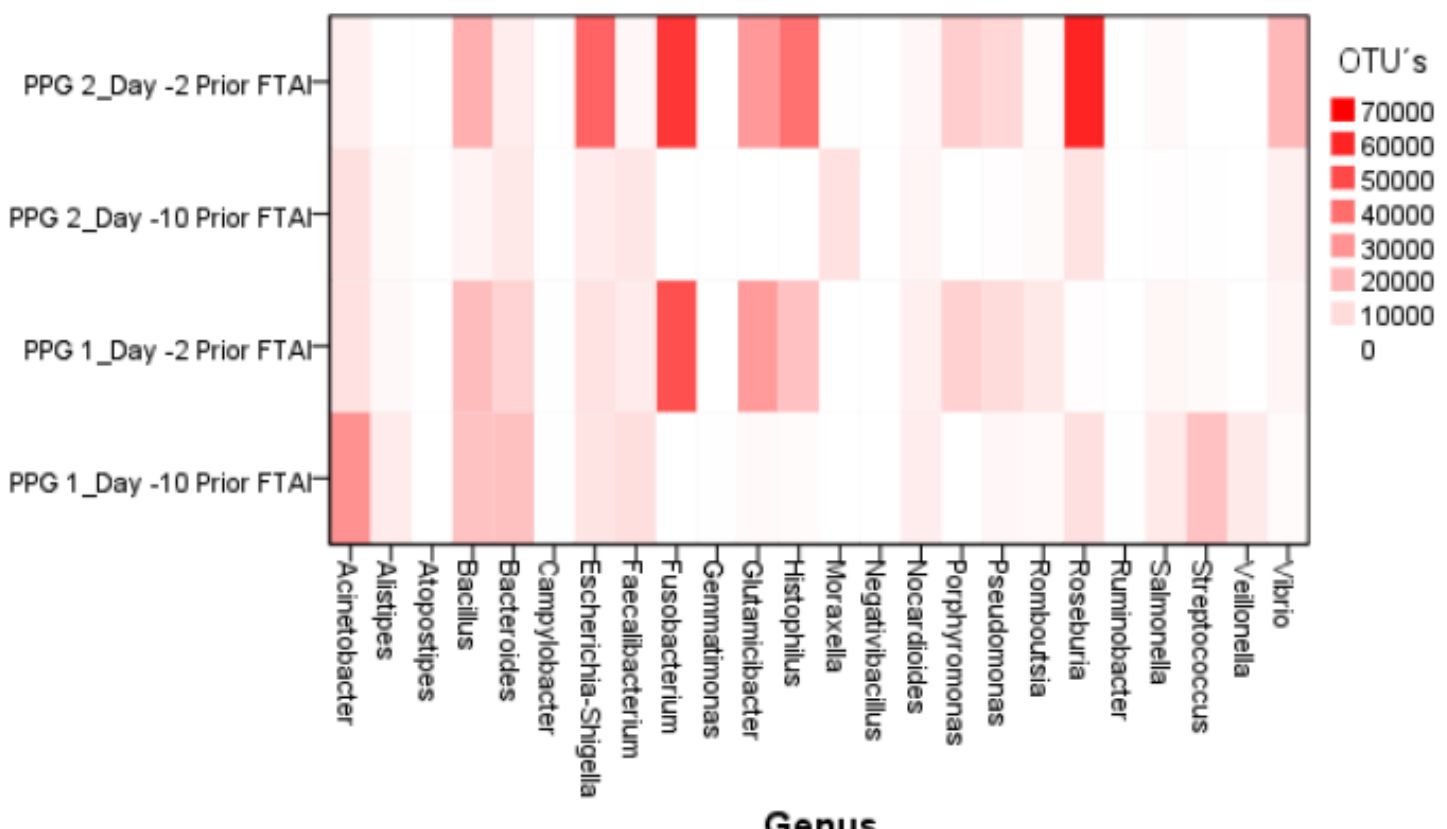

B

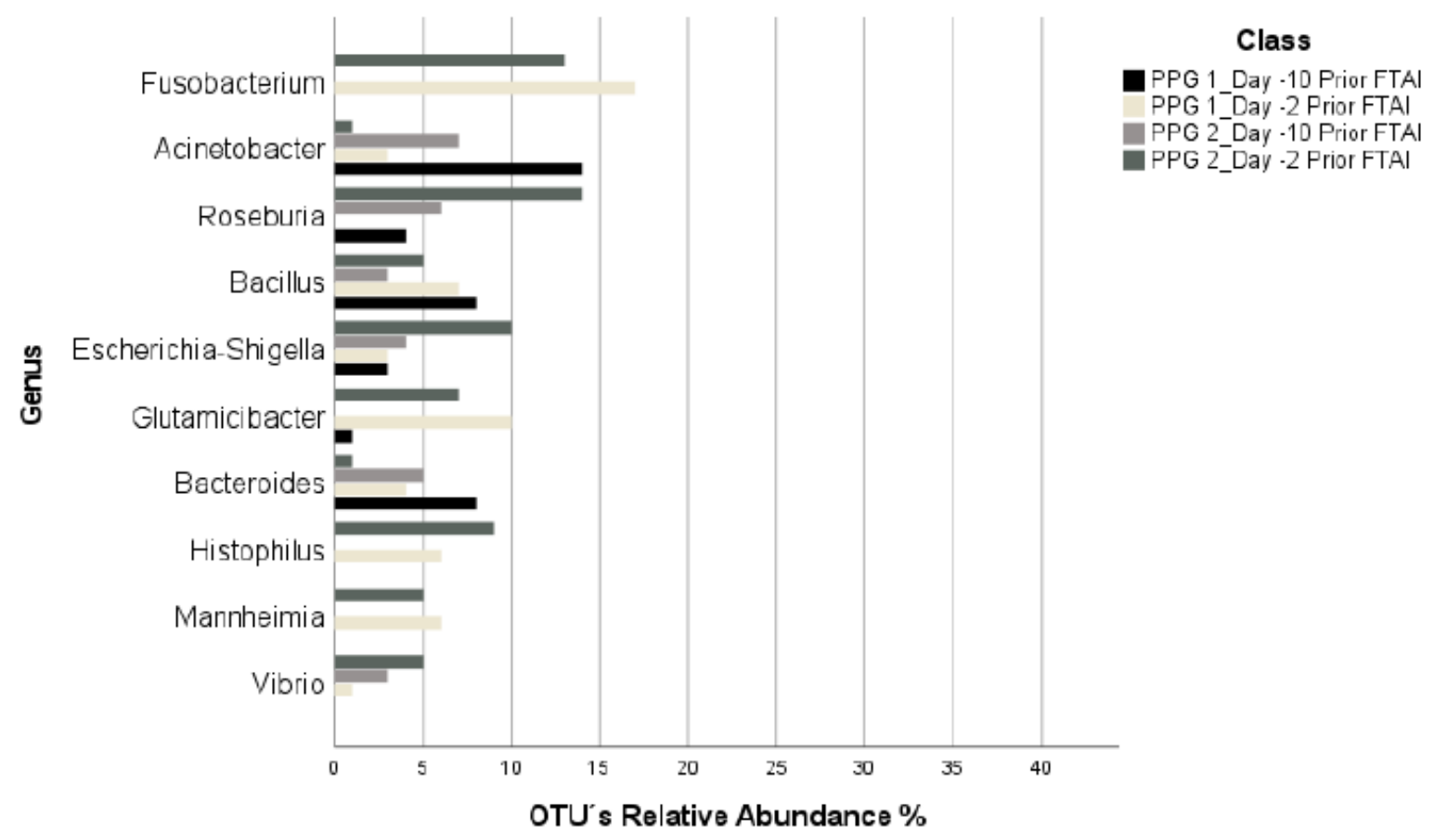

\section{Figure 5}

Relevant bacterial genus identified by NGS in beef cow's prior an FTAI. A. Heatmap of relevant genus for each group, including those significant difference was determined. B. Percentage of relative abundance of the ten most genus sequenced ordered by group 


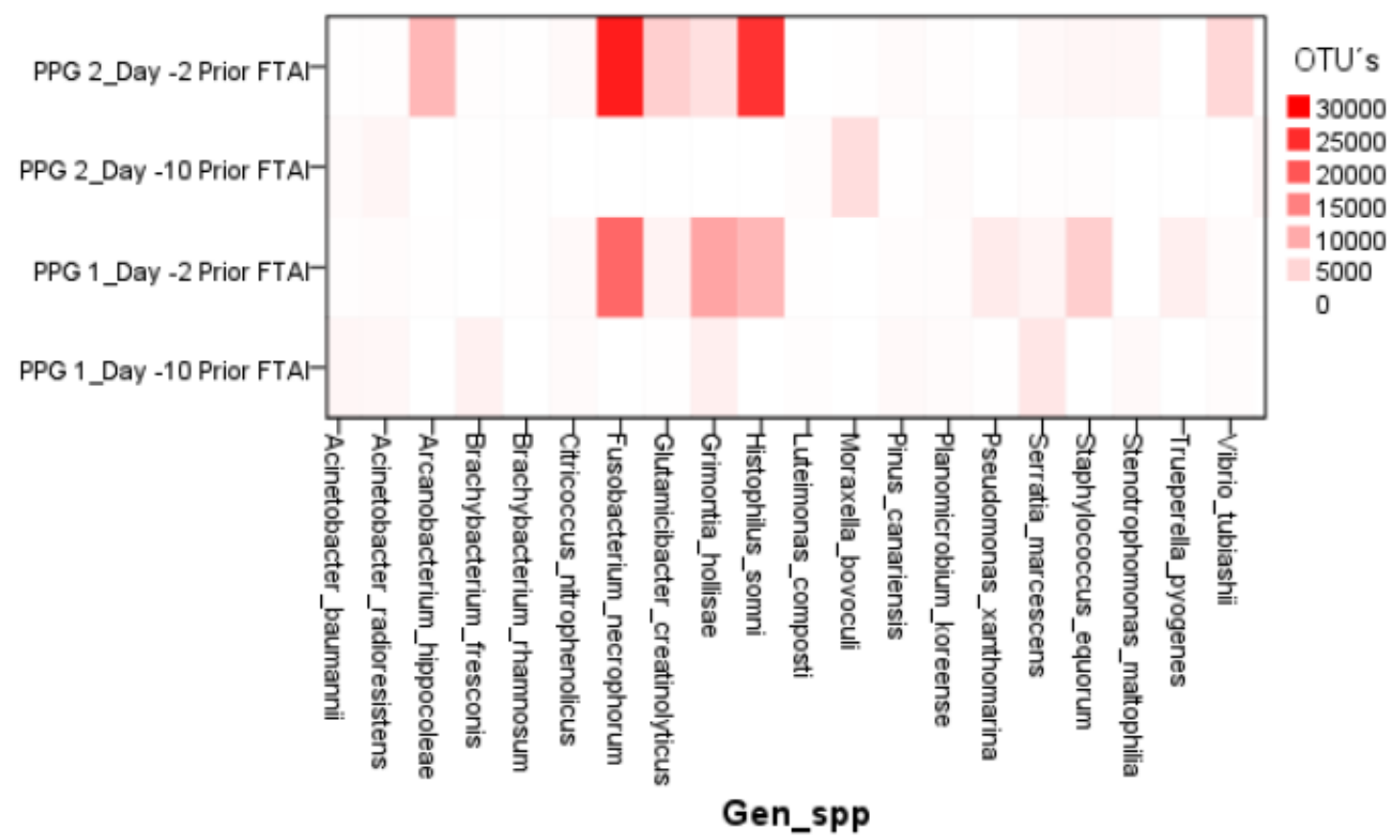

B

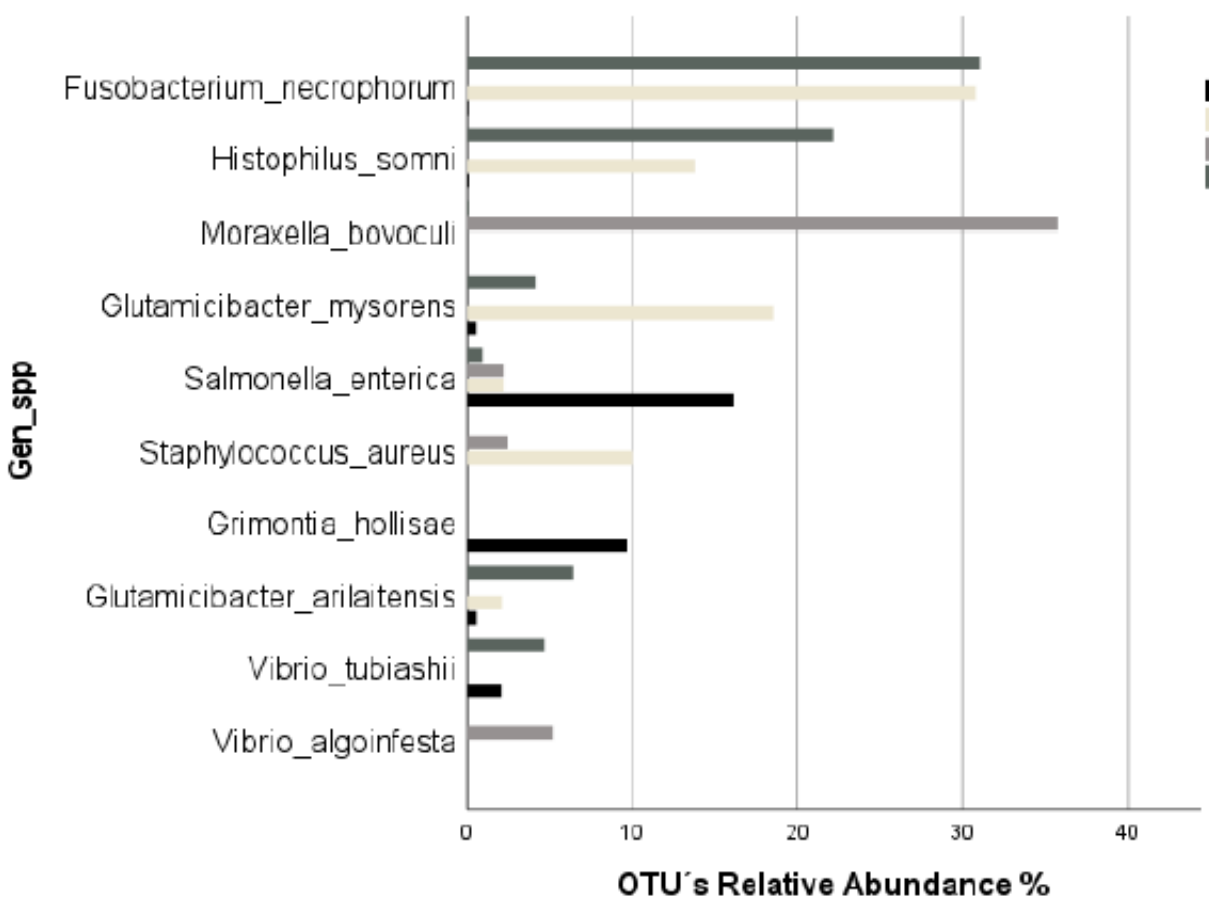

Group

PPG 1_Day - 10 Prior FTAI

PPG 1 -Day -2 Prior FTAl

PPG 2_Day - 10 Prior FTAI

PPG 2-Day -2 Prior FTAl

\section{Figure 6}

Relevant bacterial genus and species identified by NGS in beef cow's prior an FTAI. A. Heatmap of relevant genus and species for each group, including those significant difference was determined. B. Percentage of relative abundance of the ten most genus and species sequenced ordered by group 


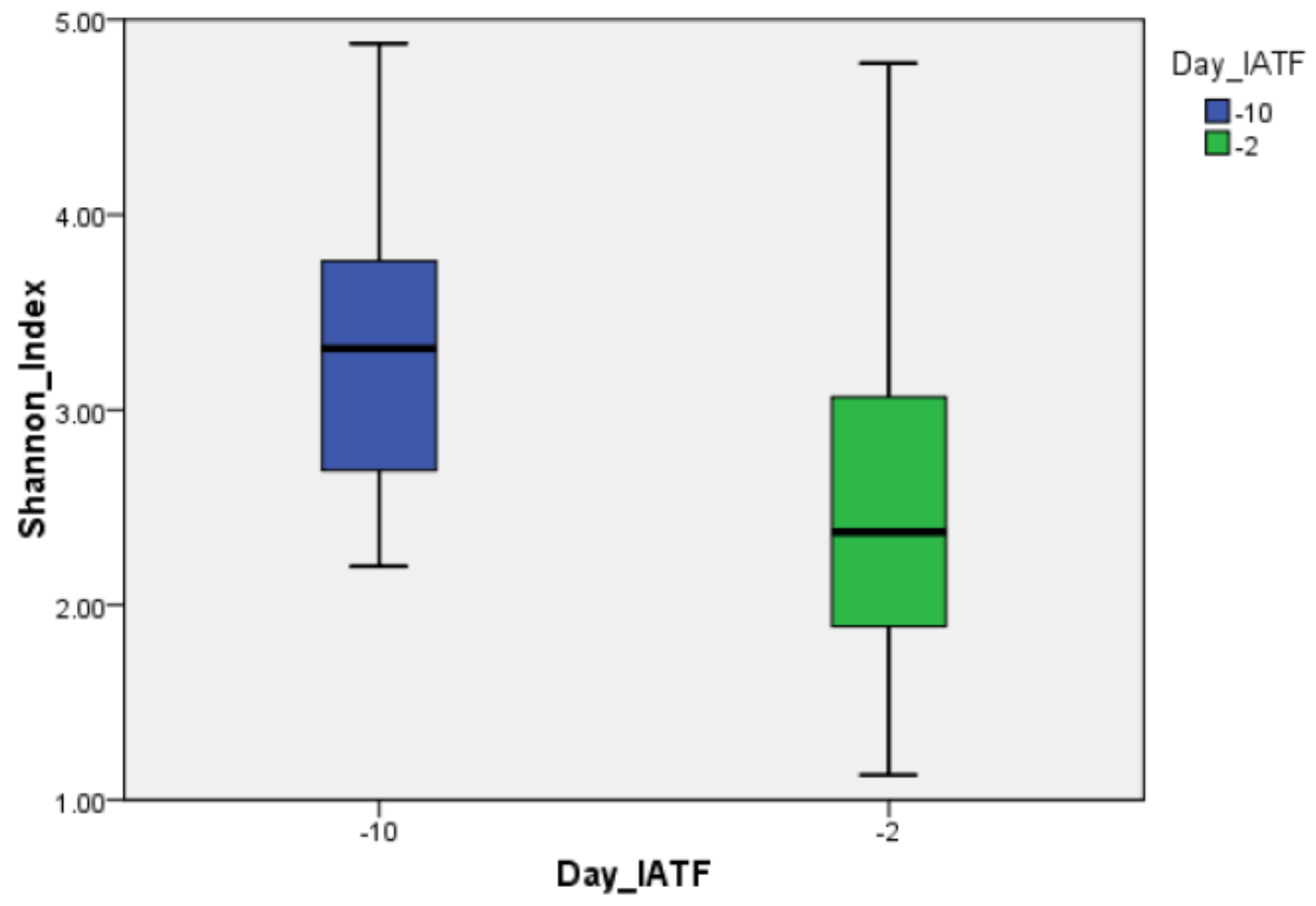

Figure 7

Comparison of Shannon's diversity in days -10 and -2 of the IATF protocol.

\section{Supplementary Files}

This is a list of supplementary files associated with this preprint. Click to download.

- SupportingDataGoogleDrive.url 